\title{
The association of community-based palliative care with reduced emergency department visits in the last year of life varies by patient factors.
}

Katrina Spilsbury $\mathrm{PhD}^{1}$, Lorna Rosenwax $\mathrm{PhD}^{2}$, Glenn Arendts $\mathrm{MBBS}^{3}$ and James B Semmens $\mathrm{PhD}^{1}$

1. Centre for Population Health Research, Faculty of Health Sciences, Curtin University, Perth,

Australia

2. School of Occupational Therapy and Social Work, Faculty of Health Sciences, Curtin

University, Perth, Australia

3. Centre for Clinical Research in Emergency Medicine, Harry Perkins Institute of Medical Research and The University of Western Australia, Perth Australia; Department of Emergency Medicine, Royal Perth Hospital, Perth, Australia.

Corresponding author: Lorna Rosenwax, Faculty of Health Sciences, Curtin University, GPO Box U1987, Perth 6845, Australia Phone:+61892667466 Fax:+61892661866

Email: L.Rosenwax@curtin.edu.au

Conflict of interest: The authors declare they have no competing interests.

Word count: 3532

Funding: This study was supported by an Australian National Health and Medical Research Council project grant (\#1029663).

Author contributions: LR and JS conceived and designed the study and obtained research funding. KS conducted the statistical analysis and drafted the manuscript. GA provided clinical interpretation of findings. The article was critically reviewed by all authors. 


\section{Abstract}

Objective: Palliative care has been shown to reduce end-of-life emergency department (ED) use. The objective was to determine how the association of community-based palliative care with reduced ED visits in the last year of life varied by patient factors.

Methods: A retrospective cohort study of 11,875 decedents who died with neoplasms, heart failure, renal failure, chronic obstructive pulmonary disease and/or liver failure in Western Australia 2009-2010. Outcome measures were adjusted hazard ratios (HR) and daily (hazard) rates of ED visits.

Results: The adjusted average rate of ED visits for the cohort was reduced $50 \%$ (HR 0.50, $95 \% \mathrm{Cl} 0.47-0.53)$ during periods of receiving community-based palliative care. This relative reduction in ED visits varied by patient factors; ranging from $43 \%$ ( $\mathrm{HR} 0.57,95 \% \mathrm{Cl} 0.45-0.74)$ for decedents aged 60 years and younger up to a $71 \%$ reduction (HR $0.29,95 \% \mathrm{Cl} 0.18-0.46$ ) for people aged 90 years and older. Decedents living in the most disadvantaged areas had a $44 \%(\mathrm{HR} 0.56,95 \% \mathrm{Cl} 0.44-0.72)$ reduction in ED visits when receiving community-based palliative care compared to a $60 \%(\mathrm{HR} 0.40,95 \% \mathrm{Cl} 0.31-0.53)$ reduction in $\mathrm{ED}$ visits for decedents who lived in the least disadvantaged areas receiving this care. The ED visit rates while receiving palliative care also varied by ED visit history, partner status and region of residence.

Conclusion: Receipt of community-based palliative care in the last year of life was associated with a reduced rate of ED visits. The magnitude of this association was modified by patient health, social and demographic factors.

Keywords: emergency department, palliative care, community-based care, population study. 


\section{Introduction}

Emergency department (ED) use increases significantly towards the end of life. ${ }^{1-4}$ Numerous studies have reported community (home)-based palliative care in people with life limiting conditions is associated with reduced use of acute care health services. ${ }^{5-9}$ While multidisciplinary palliative care has been demonstrated to reduce acute care service use, not all use of ED is unwarranted. A study of cancer patients receiving outpatient palliative care in the United States reported $77 \%$ of ED visits were unavoidable. ${ }^{10}$ However, even if most ED visits of cancer patients in the last two weeks of life result in hospital admission, it does not necessarily mean that the patient could have only been managed in the acute care setting. ${ }^{11}$ Ideally, high-quality palliative care should be able to manage the most common acute symptoms of the dying person without hospitalization.

Historically, palliative care evolved to meet the end of life needs of cancer patients. It has since become apparent that palliative care benefits non-cancer terminal conditions such as renal failure, heart failure, chronic obstructive pulmonary disease (COPD) and liver failure, although access to and quality of palliative care for these conditions could be improved. ${ }^{12-15}$ In Australia, there is evidence of this improved access to palliative care in non-cancer conditions over the last ten years. ${ }^{16}$

The objective of this study was to describe patterns of use of EDs by people in their last year of life and how this varied when receiving community-based palliative care. We also investigated whether any patient health, social and demographic factors modified the rates of ED visits while receiving community-based palliative care. 


\section{Materials and Methods}

\section{Study design}

This was a retrospective cohort study of persons aged 20 years and older who died from medical conditions considered amenable to palliative care. A linked de-identified extraction of all hospital admission records, death records, hospital ED visits and community-based care records was obtained from the Data Linkage Branch, Western Australian (WA) Department of Health. The ED presentations from 365 days prior to date of death until death (i.e. the last year of life), as well as place of residence at each hospital admission and at time of death, was available for each decedent. Ethical approval was obtained from Curtin University and the WA Department of Health Human Research Ethics Committees.

\section{Cohort selection}

The final cohort was selected from death registration records. People who died with conditions amenable to palliative care in WA from 1 January 2009 to 31 December 2010, and who were aged 20 years or greater were identified. This was achieved by searching Part 1 of the death certificate (underlying causes of death) for any mention of the most frequent conditions amenable to palliative care identified by Rosenwax et al, ${ }^{17}$ namely neoplasia, heart failure, renal failure, liver failure, chronic obstructive pulmonary disease (COPD), Alzheimer's disease, motor neurone disease, Parkinson's disease, Huntington's disease and HIV/AIDS. The underlying antecedent cause of death took precedence over more direct causes of death when more than one condition amenable to palliative care was present. ${ }^{18}$ Due to small samples sizes, decedents with motor neurone disease, Parkinson's disease, Huntington's disease and HIV/AIDS were excluded. Decedents with Alzheimer's dementia were also 
excluded because we have published a detailed analysis of ED use and palliative care in this group elsewhere. ${ }^{19}$

\section{Community-based palliative care}

Community-based palliative care was defined as the palliative care provided by the not-forprofit organisation, Silver Chain WA. ${ }^{20}$ Silver Chain WA provides over $90 \%$ of in-home palliative care in WA. Their community-based palliative care service area is largely based in the major metropolitan city of Perth where approximately $75 \%$ of the state population resides. The community-based palliative care service is a 24 hour in-home specialist nursing and, where required, medical service provided by primary care physicians and palliative care specialists. ${ }^{21}$ Care includes at-home physical care and practical support, symptom management (for example pain, nausea), counselling, a respite option and links to other community, spiritual and government services. Silver Chain also provide a palliative nurse consultancy service to public/private hospitals and residential care facilities where patient care is managed by a registered nurse 24 hours a day. A palliative rural telephone advisory service offers specialist advice and knowledge to rural service providers regarding the palliative care needs of specific patients.

Access to community-based palliative care requires referral from a medical practitioner or hospital for patients with active, progressive, terminal illness requiring symptom management. This generally occurs within three months of death but can be 12 months or more from death. It has been reported that patients with life-limiting conditions with slow or fluctuating trajectories that required longer term palliative care may have more difficulty accessing palliative care. ${ }^{22}$

Emergency department visits 
An ED visit was defined as the first presentation of the day to an ED whether it resulted in a hospital admission or not. Multiple presentations to ED on the same day were considered a single ED visit day. The ED visits that occurred as part of hospital transfers were excluded from the analysis. Available ED data included the triage category, hospital admission status and presenting symptom. Coded presenting symptom information was available for major metropolitan public hospitals only (approximately $80 \%$ of ED visits). The number of previous ED visits that had already occurred in the last year of life at the time of each ED visit was recorded.

\section{Variable definitions}

Residential address was classified as private residence, residential aged care facility (RACF), other types of care facilities or other/unknown. Marital status at time of death was classified as partnered or non-partnered. Accessibility categories were based on the ARIA+ index ${ }^{23}$ which measures accessibility to services by taking road distances to nearest service centers and population size into account. Socioeconomic status was estimated from the Index of Relative Disadvantage (IRSD) which estimates the average disadvantage of small geographic areas based on 17 measures including education, income, occupation and unemployment. ${ }^{24}$ Comorbidity was defined as the presence of one or more of the 30 Elixhauser conditions recorded during in-patient hospital stays after excluding the principal condition leading to death. ${ }^{25}$

\section{Statistical analyses}

Chi-squared tests were used to assess the equality of proportions. Linear regression models were used to assess equality of means across principal conditions using neoplasia as the reference groups. Nonparametric K-sample tests for the equality of medians were used. 
For the time to event (survival) analyses, a marginal models approach was used and multiple ED visits per decedent were accounted for using robust variance estimators. Each day in the last year of life of each decedent was assigned as no care (no hospital or community-based palliative care), in-patient hospital care or community-based palliative care

A decedent was not considered at risk of an ED admission while in hospital and was removed from the risk pool during each hospital stay (interval truncation). The Nelson-Aalen estimator of the cumulative hazard function was used to visualize the accumulated total average number of ED visits in the last year of life $\mathrm{e}^{26,27}$. As decedents could switch between receiving palliative care and then not receiving palliative care, Figure 1 does not necessarily represent the exact same group of decedents on any one day.

To easily visualize the baseline hazards, a flexible parametric proportional hazards model that used restricted cubic splines (Royston-Parmar models) was constructed. ${ }^{28}$ Time-fixed (e.g. sex) and time-varying covariates (e.g. ED history and community-based palliative care state) variables were included. Time-dependent effects, i.e. when the hazard ratio of a covariate changed over time (non-proportional hazards), were modelled as cubic splines. Interaction terms were included in the final model (Table 4) if the joint test of the two interaction terms or the test of an interesting partial interaction effect resulted in a $p$-value $<0.05$. Baseline complexity was described using seven internal knots for the main model and two internal knots for the time-dependent effects. Stata Statistical Software: Release 13 (Stata Corp, College Station, TX) was used. 


\section{Results}

There were 12817 deaths in WA from one or more of ten conditions identified as being amenable to palliative care ${ }^{17}$ over the study period (2009-2010). Of these, 11875 (93\%) decedents had one or more of the five most frequent conditions amenable to palliative care, namely neoplasm, heart failure, renal failure, COPD or liver failure. This group of decedents formed the cohort of interest. Most of the cohort ( $n=9212 ; 78 \%$ ) attended an ED at least once in the last year of life resulting in a combined total of 26,020 ED visits. The median number of ED visits per decedent was two (Interquartile range 1 -4) although there was a wide range with one decedent recording 74 ED visit days in the last year of life.

The social, demographic and health characteristics of decedents varied significantly by the number of ED visits in the last year of life (Table 1). The proportion of decedents who had three or more ED visits increased with younger age, remoteness, social disadvantage and dying with liver failure. The proportion of decedents who did not visit an ED in the last year of life was greater for females, decedents with partners and decedents dying from neoplasms. The presence of any one of the 30 Elixhauser comorbid conditions was associated with a greater proportion of ED visits compared to the cohort as a whole.

Community-based palliative care was received at least once in the last year of life by 3825 (32\%) of decedents. Almost half ( $n=3481 ; 47 \%$ ) of decedents with neoplasms received community-based palliative care compared to $13 \%(n=26)$ of decedents with liver failure, $10 \%$ $(n=110)$ with COPD, $8 \%(n=91)$ with renal failure and $6 \%(n=117)$ with heart failure. A summary of ED and hospital use in the last year of life is shown in Table 2 stratified by whether any community-based palliative care was accessed at any time in the last year of life. Decedents who accessed community-based palliative care in the last year of life tended 
towards a lower mean number of ED visits per year, a greater proportion who did not visit ED at all in the last year of life and fewer ED visits leading to hospital admissions. Decedents who received community-based palliative care presented to ED with less shortness of breath but more pain and nausea symptoms compared to other decedents. A higher proportion of decedents who received community-based palliative care were admitted to hospital for acute care more often, although, on average they stayed for shorter periods of time and spent less time in ICU.

The proportion of decedents who visited ED increased with closeness to date of death (Figure 1A). Similarly, a decedent's likelihood of receiving community-based palliative care or being admitted to hospital increased with closeness to death. Time to event analysis with interval truncation was used to describe the association of receiving community-based palliative care with ED visits because it could take both the changes due to closeness to time of death and whether a decedent is at risk of an ED visit (i.e. not in hospital) into account. The average accumulation of ED visit days in the last year of life was reduced during periods of receiving community-based palliative care compared to no palliative care (Figure 1B). The data was structured to account for when individual decedents started and stopped community-based palliative care based on their actual service dates. Thus, Figure $1 \mathrm{~B}$ is interpreted as the cumulative average number of ED visits that would accumulate if decedents continuously received or didn't receive palliative care over last year of life.

Multivariate time to event regression analysis was undertaken to determine whether the association of reduced rate of ED visits while receiving community-based palliative care remained after adjusting for patient social, demographic and health related variables (Table 3). During periods of receiving community-based palliative care, the ED visit rate was reduced 
by $50 \%(95 \% \mathrm{Cl} 47-53)$ compared to ED visit rates during periods of not accessing palliative care and after accounting for multiple other covariates. Other factors associated with reduced ED visits were older age at death, residing in a care facility, less social disadvantage, living in a major city, dying with heart failure or neoplasms, no previous history of ED visits and not having additional comorbidity.

Although community-based care was associated with an average $50 \%$ reduced rate of ED visits, we wanted to determine whether the size of the reduction was modified by patient social, demographic and health related factors. The regression model in Table 3 was tested for significant interactions between receiving community-based palliative care and the other covariates. Five significant interaction terms were observed and showed that the association of community-based palliative care with reduced ED visits varied by decedent's age, partner status, residential location, level of social disadvantage and ED history (Table 4). It was observed that receiving community-based palliative care was associated with a greater reduction in ED visits with increasing age, having a partner, living in major cities and living in areas of least social disadvantage (Table 4). Although prior history of ED visits showed some variation in hazard ratios, there was no obvious trend. There was no evidence that the association of community-based palliative care with reduced ED visit rates varied by the principal condition underlying death, decedent sex, type of residence or comorbid conditions. 


\section{Discussion}

In this large population-based study, periods of time receiving community-based palliative care in the last year of life was associated with an average $50 \%$ reduction in rate of ED visits. While a reduction in acute care service use has been consistently reported for end of life patients who receive community (home) care, both in Australia ${ }^{19,29}$ and internationally-9, 30, it is encouraging to report here that the association with reduced ED use was similar for all of the five different underlying cause of death conditions, gender and living arrangements.

The association of community-based palliative care with reduced ED use in life-limiting conditions showed significant variation amongst several other patient characteristics. While community-based palliative care was associated with a decreased rate of ED use at all ages, it appeared most effective in people aged 90 years and older. This association with a bigger reduction of ED visits by the most elderly receiving palliative care is possibly a reflection of different attitudes towards dying compared with younger decedents. A survey of older persons (average age 97 years) reported that most felt ready to die, preferred being made comfortable rather than having life-saving treatments and wished to avoid hospital ${ }^{31}$. This may not be the case for younger decedents and their caregivers. It could be argued that younger decedents tended to have different life-limiting conditions leading to different symptom management needs compared to older patients. Our data lacked sufficient symptom detail to test this, however an Italian study of people with advanced cancer in the weeks leading up to death found that there was no difference in symptoms between the age groups but that older patients required less palliative sedation. ${ }^{32}$ This supports the idea that, at least in cancer, age variation of ED use in the last year of life may be driven by both attitude and symptom management. 
The diminishing association of community-based palliative care on ED visit rate with further distances away from the major metropolitan center was not surprising as the single community-based care provider (Silver Chain WA) of this study is based in the capital city. The service decreased to only nursing care/telephone advisory services in more remote areas. The rural and remote area of WA covers 2.6 million square kilometers and was populated by just over half a million inhabitants during the study period. In addition, a separate WA government funded initiative to improve rural palliative care began implementation over $2009 / 2010^{33}$, potentially leading to some misclassification bias as decedents were increasingly able to source specialist palliative care from other sources. Despite this limitation, we were still able to detect a significant association of community-based palliative care with reduced ED visits for decedents living in rural and remote areas signifying the value of the specialist palliative care telephone advisory service provided to local health personnel. This is supported by a recent study that demonstrated professional palliative care education delivered by video conferencing to remote north Queensland (Australia) improved care provider confidence. ${ }^{34}$

Decedents living in areas of less disadvantage showed a lower rate of ED visits compared to decedents living in areas of more disadvantage during periods of receiving community-based palliative care. Decedents who lived in areas of less disadvantage were more likely to have had additional financial resources to support community-based care; this may have the effect of increasing the threshold for attending an ED. These decedents may also have higher rates of health literacy and self-advocacy that influence access to non-hospital care.

Part of the reduced rate of ED visits observed during periods of time that decedents received community-based palliative care appeared to be mediated through decedent's partners who 
were presumed to have a carer role. This suggests that the community-based palliative care service provided a level of support that enabled carers to feel they need not take their partners to ED so often. While this is an encouraging finding, further research is required to ensure the optimal carer support is being provided in light of several studies that report some caregivers still lack confidence in managing pain, experience difficulty in communicating with the palliative care team and struggle with accepting patients mortality. ${ }^{35,36}$

For the cohort as a whole, a history of previous ED use in the last year of life was associated with increased rates of further ED visits. Such an association has been reported previously with frequent ED attenders more likely to have previous hospital admission, report mental health conditions, and experience social disadvantage and reduced access to primary care. ${ }^{37}$ While receipt of community-based palliative care was also associated with a reduction in rate of ED visits in the cohort of frequent ED attenders, the reduction was of similar magnitude to those without an ED visit history. Research on how community-based palliative care programs could optimize patient confidence in home care for this patient subgroup is needed. However, a large proportion of ED visits in the last year of life resulted in hospitalization suggesting that many ED visits were appropriate. A detailed US study reported that in cancer patients receiving out-patient palliative care as many as $77 \%$ of ED visits could be classed as unavoidable. ${ }^{10}$ Simply focusing on reducing the ED visit rate further without taking different patient factors into account could be harmful in some cases.

A limitation of this study was not being able to determine the severity of the underlying cause of death. It is likely that some decedents were not considered to be at the end stage of their disease and who died earlier than might have been expected, perhaps while still receiving curative treatments, and/or in the case of organ failure hopeful of an organ transplant cure. In these cases, the motivation for attending an ED would be different to decedents who had 
reached a terminal disease phase and who had accepted the need for palliative care. Other limitations included the reliance of data from a single provider of palliative care service; the lack of personal perspectives on why decedents ultimately decided to attend ED.

The advantage of this study was the large population-based sample and use of administrative data sets that increased the generalizability of our findings and reduced institutional selection bias. The Australian health care system provides universal access to health care through a system of public and private insurance. The palliative care from Silver Chain WA was provided free of cost to the decedents giving our study the advantage of being generalizable across a wide community base. However, when interpreting these findings, each decedent's needs, expectations and experiences of community-based palliative care would have been individual and influenced by factors like age, trajectory of the underlying disease, level of family support and acceptance of their condition. ${ }^{38}$ In addition, community-based palliative care services offered in other countries may be very different to those described here.

In conclusion, we report reduced rates of ED visits during periods that decedents were receiving community-based palliative care. This reduction was consistent regardless of underlying disease and comorbidities, gender and whether a patient was living at home or in residential care. However, the magnitude of reduction in ED visits varied across decedent age groups, residential location, socioeconomic status, ED history and partner. This demonstrates the importance of considering patient social, demographic and health factors when planning community-based palliative care service delivery for the last year of life. 


\section{Acknowledgements}

The authors wish to thank the staff at the Western Australian Data Linkage Branch and the custodians of the Hospital Morbidity Data Collections, the Births, Deaths and Marriages register and the Silver Chain community care group for access to and linkage of their data.

\section{References}

1. Goldsbury DE, O'Connell DL, Girgis A, et al. Acute hospital-based services used by adults during the last year of life in New South Wales, Australia: a population-based retrospective cohort study. BMC Health Serv Res. 2015;15:537.

2. Rosenwax LK, McNamara BA, Murray K, McCabe RJ, Aoun SM, Currow DC. Hospital and emergency department use in the last year of life: a baseline for future modifications to endof-life care. Med J Aust. 2011;194:570-573.

3. Feng Z, Coots LA, Kaganova Y, Wiener JM. Hospital and ED use among Medicare beneficiaries with dementia varies by setting and proximity to death. Health Aff (Millwood). 2014;33:683690.

4. Lawrenson R, Waetford J, Gibbons V, Kirk P, Haggar S, Reddy R. Palliative care patients' use of emergency departments. The New Zealand medical journal. 2013;126:80-88.

5. Gomes B, Calanzani N, Curiale V, McCrone P, Higginson IJ. Effectiveness and cost-effectiveness of home palliative care services for adults with advanced illness and their caregivers. Cochrane Database Syst Rev. 2013;6:Cd007760.

6. Brumley RD, Enguidanos S, Cherin DA. Effectiveness of a home-based palliative care program for end-of-life. Journal of palliative medicine. 2003;6:715-724. 
7. Chan KY, Cheng HW, Yap DY, et al. Reduction of acute hospital admissions and improvement in outpatient attendance by intensified renal palliative care clinic follow-up: the Hong Kong experience. Journal of pain and symptom management. 2015;49:144-149.

8. lupati SP, Ensor BR. Do community hospice programmes reduce hospitalisation rate in patients with advanced chronic obstructive pulmonary disease? Internal medicine journal. 2016;46:295-300.

9. Seow H, Barbera L, Howell D, Dy SM. Using more end-of-life homecare services is associated with using fewer acute care services: a population-based cohort study. Med Care. 2010;48:118-124.

10. Delgado-Guay MO, Kim YJ, Shin SH, et al. Avoidable and unavoidable visits to the emergency department among patients with advanced cancer receiving outpatient palliative care. Journal of pain and symptom management. 2015;49:497-504.

11. Barbera L, Taylor C, Dudgeon D. Why do patients with cancer visit the emergency department near the end of life? Canadian Medical Association Journal. 2010;182:563-568.

12. Feyi $\mathrm{K}$, Klinger S, Pharro $\mathrm{G}$, et al. Predicting palliative care needs and mortality in end stage renal disease: use of an at-risk register. BMJ Support Palliat Care. 2015;5:19-25.

13. Potosek J, Curry M, Buss $M$, Chittenden E. Integration of palliative care in end-stage liver disease and liver transplantation. Journal of palliative medicine. 2014;17:1271-1277.

14. Vermylen JH, Szmuilowicz E, Kalhan R. Palliative care in COPD: an unmet area for quality improvement. Int J Chron Obstruct Pulmon Dis. 2015;10:1543-1551.

15. Whellan DJ, Goodlin SJ, Dickinson MG, et al. End-of-life care in patients with heart failure. J Card Fail. 2014;20:121-134.

16. Rosenwax L, Spilsbury K, McNamara B, Semmens JB. A retrospective population based cohort study of access to specialist palliative care in the last year of life: Who is still missing out a decade on? BMC Palliative Care. 2016; In press. 
17. Rosenwax LK, McNamara B, Blackmore AM, Holman CD. Estimating the size of a potential palliative care population. Palliative medicine. 2005;19:556-562.

18. Pink B. Information Paper: Cause of Death Certification, Australia. Canberra: Australian Bureau of Statistics, Commonwealth of Australia; 2008.

19. Rosenwax L, Spilsbury K, Arendts G, McNamara B, Semmens J. Community-based palliative care is associated with reduced emergency department use by people with dementia in their last year of life: A retrospective cohort study. Palliative medicine. 2015;29:727-736.

20. Silver Chain Group. Silver Chain Western Australia. Vol 20142014.

21. Group SC. SILVER CHAIN GROUP SUBMISSION ON THE CONSULTATION PAPER ON THE PRICING FRAMEWORK FOR AUSTRALIAN PUBLIC HOSPITAL SERVICES 2016-172015.

22. Department of Health WA. Palliative Care Model of Care. In: Network WCPC, ed. Perth: Department of Health, Western Australia; 2008.

23. Glover J, Tennant S. Remote Areas Statistical Geography in Australia: Notes on the Accessibility/Remoteness Index for Australia (ARIA + version). Vol Working Paper Series No. 9. Adelaide: Public Health Information Development Unit; 2003.

24. Pink B. Information Paper: An Introduction to Socio-Economic Indexes for Areas (SEIFA) 2006. Vol Cat No 2039.0. Canbera: Australia Bureau of Statistics (ABS); 2008.

25. Elixhauser A, Steiner C, Harris DR, Coffey RM. Comorbidity measures for use with administrative data. Med Care. 1998;36:8-27.

26. Aalen O. Nonparametric Inference for a Family of Counting Processes. Annals of Statistics. $1978 ; 6: 701-726$.

27. Nelson W. Theory and Applications of Hazard Plotting for Censored Failure Data. Technometrics. 1972;14:945-\&.

28. Lambert PC, Royston P. Further development of flexible parametric models for survival analysis. Stata Journal. 2009;9:265-290. 
29. McNamara BA, Rosenwax LK, Murray K, Currow DC. Early admission to community-based palliative care reduces use of emergency departments in the ninety days before death. Journal of palliative medicine. 2013;16:774-779.

30. Pouliot K, Weisse CS, Pratt DS, DiSorbo P. First-Year Analysis of a New, Home-Based Palliative Care Program Offered Jointly by a Community Hospital and Local Visiting Nurse Service. Am J Hosp Palliat Care. 2015.

31. Fleming J, Farquhar M, Cambridge City over-75s Cohort study c, Brayne C, Barclay S. Death and the Oldest Old: Attitudes and Preferences for End-of-Life Care - Qualitative Research within a Population-Based Cohort Study. PloS one. 2016;11:e0150686.

32. Mercadante S, Aielli F, Masedu F, Valenti M, Verna L, Porzio G. Age differences in the last week of life in advanced cancer patients followed at home. Supportive care in cancer : official journal of the Multinational Association of Supportive Care in Cancer. 2016;24:1889-1895.

33. McConigley R. How is palliative care being provided in rural Western Australia: An evaluation of the Rural Palliative Care Model: School of Nursing and Midwifery, Curtin University; 2012.

34. Ray RA, Fried O, Lindsay D. Palliative care professional education via video conference builds confidence to deliver palliative care in rural and remote locations. BMC Health Serv Res. 2014;14:272.

35. Chi NC, Demiris G. Family Caregivers' Pain Management in End-of-Life Care: A Systematic Review. Am J Hosp Palliat Care. 2016.

36. Phongtankuel V, Scherban BA, Reid MC, et al. Why Do Home Hospice Patients Return to the Hospital? A Study of Hospice Provider Perspectives. Journal of palliative medicine. 2016;19:5156.

37. Soril L, Leggett LE, Lorenzetti DL, Noseworthy TW, Clement FM. Characteristics of frequent users of the emergency department in the general adult population: A systematic review of international healthcare systems. Health Policy. 2016. 
38. Department of Health WA. Palliative Care in Western Australia, Final Report December 2005. In: Group PCA, ed. Perth: Department of Health, Western Australia; 2005. 

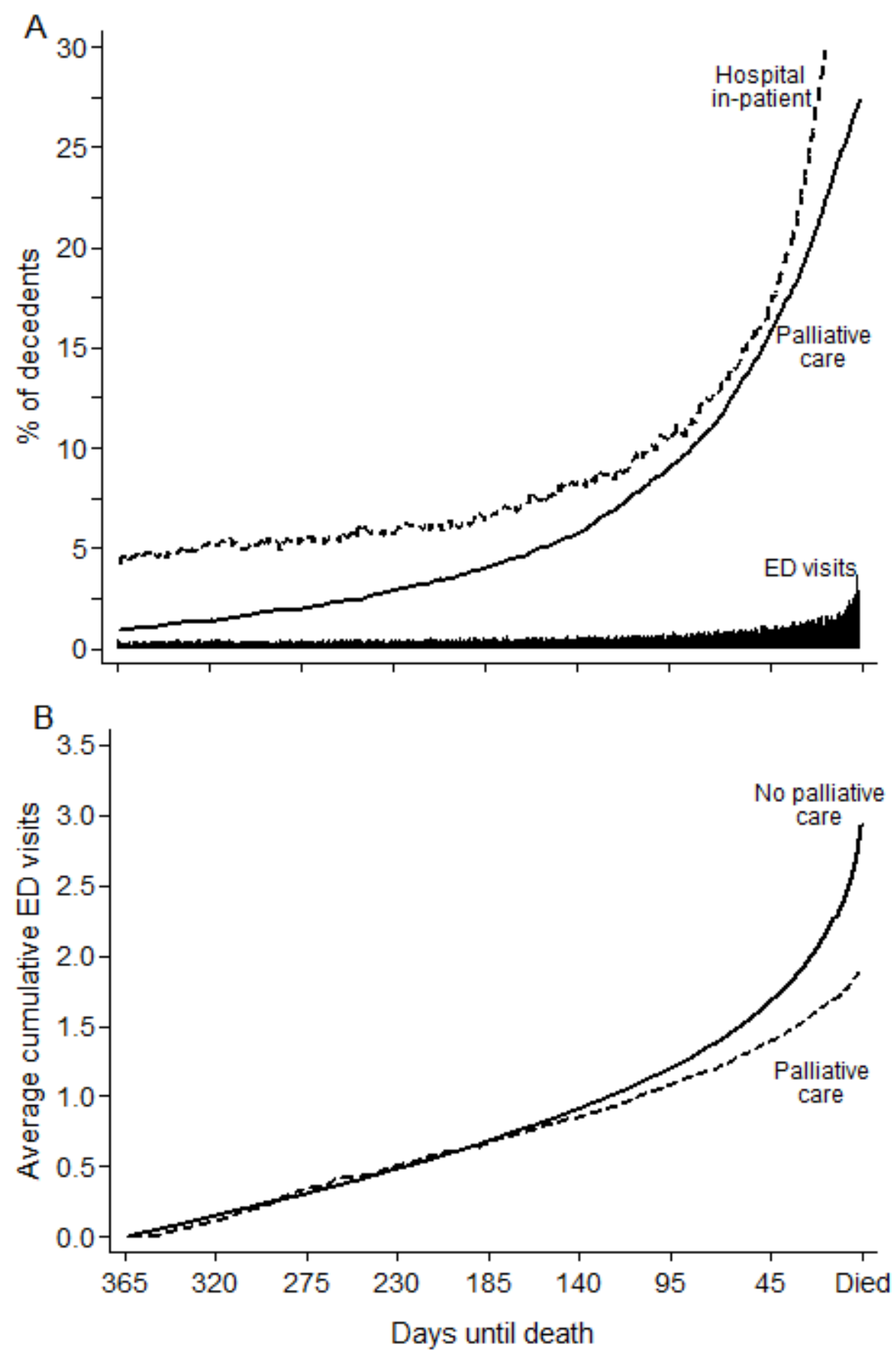
Table 1. Total number and percentage (row) of decedents in categories of ED visits in the last year of life by sociodemographic and health characteristics $(N=11,875)$.

\begin{tabular}{|c|c|c|c|c|c|c|c|c|}
\hline & \multicolumn{8}{|c|}{ Number of ED visits during last year of life } \\
\hline & \multicolumn{2}{|c|}{ None } & \multicolumn{2}{|c|}{ One } & \multicolumn{2}{|c|}{ Two } & \multicolumn{2}{|c|}{ Three or more } \\
\hline & $\mathrm{N}$ & $\%$ & $\mathrm{~N}$ & $\%$ & $\mathrm{~N}$ & $\%$ & $\mathrm{~N}$ & $\%$ \\
\hline Overall & 2663 & 22.4 & 3180 & 26.7 & 2188 & 18.4 & 3844 & 32.4 \\
\hline \multicolumn{9}{|l|}{ Age at death } \\
\hline$<60$ & 334 & 20.6 & 350 & 21.6 & 267 & 16.5 & 669 & 41.3 \\
\hline $60-69$ & 463 & 23.8 & 501 & 25.7 & 345 & 17.7 & 639 & 32.8 \\
\hline $70-79$ & 629 & 20.9 & 845 & 28.1 & 537 & 17.8 & 998 & 33.2 \\
\hline $80-89$ & 830 & 21.8 & 1087 & 28.6 & 736 & 19.4 & 1149 & 30.2 \\
\hline $90+$ & 407 & 27.2 & 397 & 26.5 & 303 & 20.3 & 389 & 26.0 \\
\hline \multicolumn{9}{|l|}{ Sex } \\
\hline Male & 1421 & 21.8 & 1677 & 25.7 & 1171 & 17.9 & 2260 & 34.6 \\
\hline Female & 1242 & 23.2 & 1503 & 28.1 & 1017 & 19.0 & 1584 & 29.6 \\
\hline \multicolumn{9}{|l|}{ Partnered at death } \\
\hline No & 1333 & 21.1 & 1758 & 27.8 & 1185 & 18.8 & 2037 & 32.3 \\
\hline Yes & 1330 & 23.9 & 1422 & 25.6 & 1003 & 18.0 & 1807 & 32.5 \\
\hline \multicolumn{9}{|l|}{ Accessibility index } \\
\hline Major cities & 2030 & 25.2 & 2251 & 27.9 & 1477 & 18.3 & 2302 & 28.6 \\
\hline Inner regional & 338 & 17.3 & 520 & 26.6 & 390 & 19.9 & 707 & 36.2 \\
\hline Outer regional & 195 & 16.9 & 272 & 23.6 & 208 & 18.1 & 477 & 41.4 \\
\hline Remote & 59 & 14.4 & 80 & 19.5 & 72 & 17.5 & 200 & 48.7 \\
\hline Very remote & 29 & 13.1 & 37 & 16.7 & 27 & 12.2 & 129 & 58.1 \\
\hline Unknown & - & - & 9 & 18.8 & 10 & 20.8 & 27 & 56.3 \\
\hline
\end{tabular}

Index relative social disadvantage

$\begin{array}{rrrrrrrrr}\text { Most disadvantaged } & 394 & 15.2 & 633 & 24.4 & 499 & 19.2 & 1072 & 41.3 \\ \text { More disadvantaged } & 477 & 18.5 & 685 & 26.6 & 521 & 20.2 & 897 & 34.8 \\ \text { Average disadvantage } & 528 & 21.9 & 652 & 27.0 & 461 & 19.1 & 774 & 32.0 \\ \text { Less disadvantage } & 495 & 24.7 & 561 & 28.0 & 355 & 17.7 & 590 & 29.5 \\ \text { Least disadvantage } & 757 & 34.3 & 628 & 28.5 & 339 & 15.4 & 483 & 21.9 \\ \text { Unknown } & - & - & 9 & 19.6 & 9 & 19.6 & 26 & 56.5 \\ & & & & & & & & \\ \text { Residence at death } & & & & & & & & \\ \text { Private residence } & 2122 & 22.3 & 2518 & 26.5 & 1749 & 18.4 & 3121 & 32.8 \\ \text { Residential aged care } & 479 & 22.8 & 603 & 28.7 & 387 & 18.4 & 632 & 30.1 \\ \text { Other care facility } & 35 & 27.6 & 26 & 20.5 & 24 & 18.9 & 42 & 33.1 \\ \text { Unknown/other/NFA } & 23 & 39.7 & 11 & 19.0 & 7 & 12.1 & 17 & 29.3\end{array}$

Principal condition underlying death

$\begin{array}{lllllllll}\text { Neoplasms } & 1840 & 24.8 & 1981 & 26.7 & 1384 & 18.7 & 2206 & 29.8\end{array}$ 


\begin{tabular}{|c|c|c|c|c|c|c|c|c|}
\hline & \multicolumn{8}{|c|}{ Number of ED visits during last year of life } \\
\hline & \multicolumn{2}{|c|}{ None } & \multicolumn{2}{|c|}{ One } & \multicolumn{2}{|c|}{ Two } & \multicolumn{2}{|c|}{ Three or more } \\
\hline & $\mathrm{N}$ & $\%$ & $\mathbf{N}$ & $\%$ & $\mathbf{N}$ & $\%$ & $\mathrm{~N}$ & $\%$ \\
\hline Heart failure & 395 & 19.6 & 560 & 27.7 & 378 & 18.7 & 686 & 34.0 \\
\hline Renal failure & 205 & 17.9 & 285 & 24.9 & 227 & 19.8 & 428 & 37.4 \\
\hline Chronic obstructive pulmonary disease & 202 & 18.5 & 296 & 27.1 & 177 & 16.2 & 419 & 38.3 \\
\hline Liver failure & 21 & 10.2 & 58 & 28.2 & 22 & 10.7 & 105 & 51.0 \\
\hline \multicolumn{9}{|l|}{ Elixhauser Comorbid conditions ${ }^{1}$} \\
\hline Metastatic Cancer & 33 & 15.4 & 55 & 25.7 & 41 & 19.2 & 85 & 39.7 \\
\hline Rheumatoid Arthritis/Collagen Vascular & 24 & 15.3 & 34 & 21.7 & 31 & 19.7 & 68 & 43.3 \\
\hline Liver Disease & 81 & 14.4 & 138 & 24.5 & 95 & 16.9 & 249 & 44.2 \\
\hline Solid Tumor Without Metastasis & 58 & 14.3 & 101 & 24.9 & 81 & 20.0 & 166 & 40.9 \\
\hline Depression & 91 & 14.2 & 146 & 22.8 & 121 & 18.9 & 281 & 44.0 \\
\hline Pulmonary Circulation Disorders & 118 & 12.8 & 211 & 23.0 & 179 & 19.5 & 411 & 44.7 \\
\hline Paralysis & 73 & 11.8 & 158 & 25.6 & 136 & 22.0 & 250 & 40.5 \\
\hline Renal Failure & 178 & 11.7 & 343 & 22.6 & 301 & 19.9 & 693 & 45.7 \\
\hline Coagulopathy & 159 & 11.7 & 308 & 22.6 & 255 & 18.7 & 640 & 47.0 \\
\hline Peripheral Vascular Disorders & 100 & 11.7 & 203 & 23.8 & 155 & 18.2 & 394 & 46.2 \\
\hline Other Neurological Disorders & 107 & 11.4 & 209 & 22.2 & 198 & 21.1 & 426 & 45.3 \\
\hline Cardiac Arrhythmias & 341 & 11.3 & 769 & 25.5 & 617 & 20.4 & 1291 & 42.8 \\
\hline Fluid and Electrolyte Disorders & 478 & 10.7 & 1083 & 24.3 & 888 & 19.9 & 2003 & 45.0 \\
\hline Diabetes, Uncomplicated & 56 & 10.7 & 121 & 23.0 & 99 & 18.9 & 249 & 47.4 \\
\hline Peptic Ulcer Disease Excluding Bleeding & 15 & 10.7 & 29 & 20.7 & 22 & 15.7 & 74 & 52.9 \\
\hline Diabetes, Complicated & 207 & 10.3 & 463 & 23.1 & 413 & 20.6 & 925 & 46.1 \\
\hline Weight Loss & 68 & 10.2 & 186 & 27.8 & 137 & 20.5 & 278 & 41.6 \\
\hline Congestive Heart Failure & 152 & 9.8 & 357 & 23.0 & 298 & 19.2 & 743 & 47.9 \\
\hline Psychoses & 7 & 9.7 & 15 & 20.8 & 15 & 20.8 & 35 & 48.6 \\
\hline Drug Abuse & 9 & 9.6 & 19 & 20.2 & 11 & 11.7 & 55 & 58.5 \\
\hline Hypertension, Uncomplicated & 334 & 9.4 & 875 & 24.5 & 734 & 20.6 & 1628 & 45.6 \\
\hline Valvular Disease & 61 & 9.1 & 165 & 24.5 & 127 & 18.9 & 320 & 47.5 \\
\hline Deficiency Anemia & 52 & 8.9 & 128 & 21.9 & 106 & 18.1 & 299 & 51.1 \\
\hline Chronic Pulmonary Disease & 105 & 8.7 & 262 & 21.7 & 261 & 21.6 & 580 & 48.0 \\
\hline Hypertension, Complicated & 13 & 8.6 & 31 & 20.4 & 32 & 21.1 & 76 & 50.0 \\
\hline Hypothyroidism & 11 & 8.4 & 23 & 17.6 & 29 & 22.1 & 68 & 51.9 \\
\hline Lymphoma & - & - & 12 & 24.5 & - & - & 26 & 53.1 \\
\hline Blood Loss Anemia & 21 & 7.7 & 49 & 18.0 & 43 & 15.8 & 159 & 58.5 \\
\hline Obesity & 17 & 6.4 & 58 & 21.9 & 48 & 18.1 & 142 & 53.6 \\
\hline Alcohol Abuse & 24 & 5.3 & 105 & 23.0 & 71 & 15.6 & 256 & 56.1 \\
\hline
\end{tabular}

${ }^{1}$ Decedents with more than one Elixhauser comorbid condition were counted multiple times. HIV/AIDs category was excluded due to low numbers. Missing value represent cells with five or less decedents. 
Table 2: Summary of health care service use in last year of life stratified by whether decedents accessed community-based palliative care at any time in the last year of life $(\mathrm{N}=11,875)$.

\begin{tabular}{|c|c|c|c|c|c|c|}
\hline \multirow{2}{*}{$\frac{\text { In last year of life }}{\text { ED visits }}$} & \multicolumn{2}{|c|}{$\begin{array}{c}\text { No Palliative care } \\
n=8050\end{array}$} & \multicolumn{2}{|c|}{$\begin{array}{c}\text { Palliative care } \\
n=3825\end{array}$} & \multicolumn{2}{|c|}{$\begin{array}{c}\text { Total } \\
\mathrm{N}=11875\end{array}$} \\
\hline & & & & & & \\
\hline \multicolumn{7}{|l|}{$\mathrm{N}(\%)$ ED visits per decedent } \\
\hline None & 1624 & 20.2 & 1039 & 27.2 & 2663 & 22.4 \\
\hline One & 2229 & 27.7 & 951 & 24.9 & 3180 & 26.8 \\
\hline Two & 1507 & 18.7 & 681 & 17.8 & 2188 & 18.4 \\
\hline Three or more & 2690 & 33.4 & 1154 & 30.2 & 3844 & 32.4 \\
\hline Mean (SD) ED visits ${ }^{1}$ & 2.9 & 2.8 & 2.7 & 2.2 & 2.8 & 2.6 \\
\hline Median (IQR) ED visits ${ }^{1}$ & 2 & $1-4$ & 2 & $1-3$ & 2 & $1-4$ \\
\hline \multicolumn{7}{|l|}{$\underline{\mathrm{N}(\%) \text { ED visits by triage category }}$} \\
\hline Resuscitation: immediate & 648 & 3.3 & 122 & 1.5 & 770 & 2.8 \\
\hline Emergency: within 10 minutes & 3744 & 18.9 & 1483 & 18.7 & 5227 & 18.9 \\
\hline Urgent: within 30 minutes & 8365 & 42.2 & 4062 & 51.3 & 12427 & 44.8 \\
\hline Semi-urgent: within 60 minutes & 5807 & 29.3 & 2057 & 26.0 & 7864 & 28.4 \\
\hline Non-urgent: within 120 minutes & 1235 & 6.2 & 192 & 2.4 & 1427 & 5.1 \\
\hline \multicolumn{7}{|l|}{$\underline{\text { Frequent ED presenting symptoms }} \underline{2}$} \\
\hline $\mathrm{N}(\%)$ with Shortness of breath & 2131 & 16.6 & 790 & 11.4 & 2921 & 14.8 \\
\hline $\mathrm{N}(\%)$ with Abdominal pain & 813 & 6.3 & 710 & 10.3 & 1523 & 7.7 \\
\hline $\mathrm{N}(\%)$ with Chest pain & 1024 & 8.0 & 488 & 7.1 & 1512 & 7.7 \\
\hline $\mathrm{N}(\%)$ with Nausea & 295 & 2.3 & 277 & 4.0 & 572 & 2.9 \\
\hline $\mathrm{N}(\%)$ with Back pain & 282 & 2.2 & 227 & 3.3 & 509 & 2.6 \\
\hline $\mathrm{N}(\%)$ ED visits led to admission² & 14608 & 73.8 & 5689 & 71.9 & 20297 & 73.2 \\
\hline \multicolumn{7}{|l|}{ Acute care hospital stays } \\
\hline $\mathrm{N}(\%)$ Individuals admitted & 7167 & 89.0 & 3608 & 94.3 & 10775 & 90.7 \\
\hline Mean (SD) no. of admissions ${ }^{1}$ & 7.6 & 17.9 & 9.1 & 12.4 & 8.1 & 16.3 \\
\hline Median (IQR) no. of admissions ${ }^{1}$ & 3 & $2-6$ & 5 & $3-12$ & 4 & $2-8$ \\
\hline Mean (SD) LOS in hospital ${ }^{1}$ & 10.0 & 13.3 & 8.6 & 10.1 & 9.5 & 12.3 \\
\hline Median (IQR) LOS in hospital ${ }^{1}$ & 6 & $2-12$ & 5 & $2-11$ & 5 & $2-12$ \\
\hline Mean(SD) days in ICU per decedent ${ }^{1}$ & 0.6 & 3.5 & 0.2 & 1.6 & 0.5 & 3.0 \\
\hline
\end{tabular}

$\mathrm{IQR}=$ inter-quartile range; $\mathrm{ED}=$ emergency department; $\mathrm{SD}=$ standard deviation; $\mathrm{LOS}=$ length of stay (days); ICU=intensive care unit

1. Estimated after excluding those decedents who had zero days of the health service in question.

2. Coded ED presenting symptoms were only available for $80 \%$ of ED visits. The five most frequent presenting symptoms overall are shown. Percentages were calculated using the number of ED visits with a symptom coded as denominator. 
Table 3: Factors associated with the rate of visits to ED in the last year of life as estimated from a multivariate main effects time-to-event regression model with time-varying and time dependent covariates.

\begin{tabular}{crcc}
\hline & HR & $\mathbf{9 5 \%} \mathbf{C l}$ \\
\hline Community-based palliative care $^{1}$ & No & 1.00 & ref \\
Age at death (years) & 0.50 & $0.47-0.53$ \\
& $<60$ & 1.00 & ref \\
& $60-69$ & 0.83 & $0.78-0.88$ \\
& $70-79$ & 0.77 & $0.73-0.81$ \\
& $80-89$ & 0.73 & $0.69-0.77$ \\
Sex & $90+$ & 0.71 & $0.67-0.76$ \\
& & & \\
Partnered at death & Male & 1.00 & ref \\
& Female & 0.96 & $0.93-0.99$ \\
& & & \\
Accessibility index & No & 1.00 & ref \\
& Yes & 0.96 & $0.93-0.99$ \\
& & & \\
& Major cities & 1.00 & ref \\
& Inner regional & 1.11 & $1.06-1.15$ \\
& Outer regional & 1.19 & $1.13-1.25$ \\
Remote & 1.36 & $1.24-1.48$ \\
& Very remote & 1.38 & $1.23-1.55$
\end{tabular}

$\begin{array}{rrcc}\begin{array}{r}\text { Index of Relative Social } \\ \text { Disadvantage }\end{array} & \text { Most disadvantaged } & 1.00 & \text { ref } \\ & \text { More disadvantaged } & 0.93 & 0.90-0.97 \\ \text { Average disadvantage } & 0.92 & 0.88-0.96 \\ & \text { Less disadvantage } & 0.90 & 0.85-0.94 \\ \text { Least disadvantage } & 0.80 & 0.76-0.84 \\ & & & \\ \text { Residence at death } & \text { Private residence } & 1.00 & \text { ref } \\ & \text { Residential aged care } & 0.68 & 0.65-0.71 \\ & \text { Other care facility } & 0.84 & 0.75-0.94 \\ \text { Principal condition }{ }^{2} & & & \\ & \text { Neoplasms } & 1.00 & \text { ref } \\ \text { Heart failure } & 0.97 & 0.93-1.02 \\ \text { Renal failure } & 1.07 & 1.01-1.14 \\ \text { COPD } & 1.27 & 1.19-1.36 \\ \text { Liver failure } & 1.39 & 1.24-1.55\end{array}$




\begin{tabular}{rcc}
\hline & HR & 95\% Cl \\
\hline Pulmonary circulatory dis. & 1.08 & $1.03-1.13$ \\
Peripheral vascular dis. & 1.10 & $1.04-1.15$ \\
Hypertension & 1.25 & $1.20-1.29$ \\
Neurological dis. & 1.21 & $1.15-1.27$ \\
Chronic pulmonary dis. & 1.21 & $1.16-1.27$ \\
Renal failure & 1.09 & $1.04-1.13$ \\
Peptic ulcer disease & 1.17 & $1.05-1.30$ \\
Metastatic cancer & 1.16 & $1.04-1.29$ \\
Coagulopathy & 1.16 & $1.11-1.21$ \\
Weight loss & 1.13 & $1.06-1.19$ \\
Fluid \& electrolyte dis. & 1.32 & $1.28-1.36$ \\
Depression & 1.22 & $1.14-1.31$
\end{tabular}

History of ED visits ${ }^{1,2}$

$\begin{array}{rcc}\text { None } & 1.00 & \text { ref } \\ \text { One } & 2.10 & 1.99-2.20 \\ \text { Two } & 2.85 & 2.63-3.09 \\ \text { Three } & 3.65 & 3.24-4.12 \\ \text { or more } & 6.81 & 5.72-8.10\end{array}$

$\mathrm{HR}=\overline{\text { hazard ratio; } \mathrm{ED}=\text { emergency department; } \mathrm{COPD}=\text { chronic obstructive pulmonary }}$ disease; dis. $=$ disease

1. Entered into model as a time-varying covariate that indicated when decedents changed states (type of care or ED history) over the last year of life.

2. Entered as time dependent variable because HRs varied over the last year of life so the single $\mathrm{HR}$ presented is an overall representation of this variation. 
Table 4. Relative rates of ED visits (hazard ratios) for periods of time receiving community-based palliative care compared to periods of time not receiving palliative care within subgroups of age, partner status, location of residence, level of disadvantage and ED visit history.

\begin{tabular}{|c|c|c|}
\hline & \multicolumn{2}{|c|}{ Palliative care versus no palliative care (ref) } \\
\hline & HR & $95 \% \mathrm{Cl}$ \\
\hline \multicolumn{3}{|l|}{ Age at death } \\
\hline$<60$ years & 0.57 & $0.45-0.73$ \\
\hline $60-69$ years & 0.58 & $0.45-0.74$ \\
\hline 70-79 years & 0.56 & $0.45-0.72$ \\
\hline $80-89$ years & 0.53 & $0.41-0.68$ \\
\hline$>=90$ years & 0.29 & $0.18-0.46$ \\
\hline \multicolumn{3}{|l|}{ Partnered } \\
\hline No & 0.53 & $0.42-0.67$ \\
\hline Yes & 0.45 & $0.35-0.58$ \\
\hline \multicolumn{3}{|l|}{ Residential location } \\
\hline Major cities & 0.43 & $0.38-0.47$ \\
\hline Inner regional & 0.55 & $0.45-0.68$ \\
\hline Outer regional & 0.49 & $0.40-0.62$ \\
\hline Remote & 0.73 & $0.54-1.00$ \\
\hline Very remote & 0.33 & $0.12-0.93$ \\
\hline \multicolumn{3}{|l|}{ Index of Relative Social Disadvantage } \\
\hline Most disadvantaged & 0.56 & $0.44-0.72$ \\
\hline More disadvantaged & 0.52 & $0.41-0.68$ \\
\hline Average disadvantage & 0.49 & $0.38-0.65$ \\
\hline Less disadvantage & 0.48 & $0.37-0.63$ \\
\hline Least disadvantage & 0.40 & $0.31-0.53$ \\
\hline \multicolumn{3}{|l|}{ ED visit history² } \\
\hline No previous visits & 0.52 & $0.40-0.67$ \\
\hline One previous visit & 0.44 & $0.34-0.57$ \\
\hline Two previous visits & 0.48 & $0.37-0.62$ \\
\hline Three previous visits & 0.58 & $0.44-0.76$ \\
\hline Four + previous visits & 0.44 & $0.34-0.58$ \\
\hline
\end{tabular}

1. Hazard ratios (HR) were estimated from a model identical to that in Table 3 except that five interaction terms between community-based palliative and the five variables shown here were included. HR for the other covariates included in this model did not vary significantly from those in Table 3 and are therefore not shown again here.

2. ED history was entered into the model as a time-dependent time-varying covariate. ref $=$ referent group against which palliative care was compared. 